\title{
JFM RAPIDS
} journals.cambridge.org/rapids

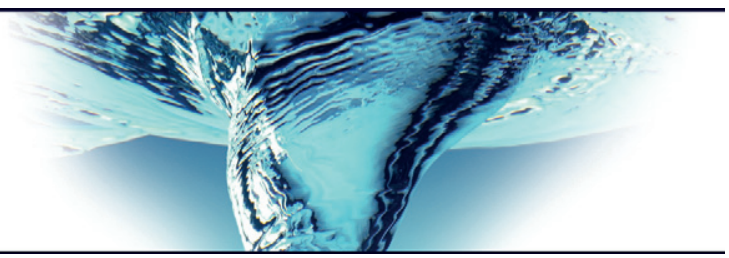

\section{A note on acoustic turbulence}

\author{
Erik Lindborg $\dagger$ \\ Department of Mechanics, KTH, SE-100 44 Stockholm, Sweden
}

(Received 12 March 2019; revised 21 June 2019; accepted 21 June 2019;

first published online 18 July 2019)

We consider a three-dimensional acoustic field of an ideal gas in which all entropy production is confined to weak shocks and show that similar scaling relations hold for such a field as for forced Burgers turbulence, where the shock amplitude scales as $(\epsilon d)^{1 / 3}$ and the $p$ th-order structure function scales as $(\epsilon d)^{p / 3} r / d, \epsilon$ being the mean energy dissipation per unit mass, $d$ the mean distance between the shocks and $r$ the separation distance. However, for the acoustic field, $\epsilon$ should be replaced by $\epsilon+\chi$, where $\chi$ is associated with entropy production due to heat conduction. In particular, the third-order longitudinal structure function scales as $\left\langle\delta u_{r}^{3}\right\rangle=-C(\epsilon+\chi) r$, where $C$ takes the value $12 / 5(\gamma+1)$ in the weak shock limit, $\gamma=c_{p} / c_{v}$ being the ratio between the specific heats at constant pressure and constant volume.

Key words: shock waves, turbulence theory, compressible turbulence

\section{Introduction}

A random acoustic field in which many modes are excited may be characterised as acoustic turbulence. A long-standing problem in turbulence theory is to determine the statistical properties of such a field. Two approaches have been developed to tackle this problem. Zakharov \& Sagdeev (1970) used weak turbulence theory to derive an energy wavenumber spectrum of the form $\sim k^{-3 / 2}$ in three dimensions. This approach was criticised by Kadomtsev \& Petviashvili (1973), who argued that shocks will always develop in an acoustic field if the Reynolds number is sufficiently large, and give rise to an energy spectrum of the form $\sim k^{-2}$. Insightful discussions on the arguments in favour of each of the two approaches are given by Falkovich \& Meyer (1996) and L'vov et al. (1997). In this note, we will not add to these discussions. Instead, we will assume that there exists a regime in which nonlinearities are sufficiently strong for shocks to form in such an abundance that virtually all entropy production is confined to shocks, and yet sufficiently weak for the shocks to be weak. The aim of the paper is to derive scaling relations for such a hypothetical regime, which may be tested experimentally or numerically. Before doing this, it

$\dagger$ Email address for correspondence: erikl@mech.kth.se 


\section{E. Lindborg}

may be illustrative to consider forced Burgers turbulence as a simple example of shock-dominated turbulence.

We consider statistically stationary solutions to Burgers equation (Burgers 1948),

$$
\frac{\partial u}{\partial t}+u \frac{\partial u}{\partial x}=v \frac{\partial^{2} u}{\partial x^{2}}+f,
$$

in a domain with length $L$, where $f$ is a random forcing with characteristic length scale $L_{f}$ and $v$ is the kinematic viscosity. Building on previous investigations it can be shown that apart from $v$ the two other parameters characterising forced Burgers turbulence are the mean energy dissipation rate per unit mass, $\epsilon$, and the mean distance between the shocks, $d=L / n$, where $n$ is the number of shocks. We define the $p$ th-order velocity structure function as $\left\langle|\delta u|^{p}\right\rangle$, where $\delta u=u(x+r)-u(x)$ and \langle\rangle is the domain average. If $p$ is an odd integer we can also define structure functions without taking the absolute value of $\delta u$ - for example, the third-order structure function $\left\langle\delta u^{3}\right\rangle$. As shown by Bouchaud, Mezard \& Parisi (1995), Weinan et al. (1997) and Weinan \& Eijnden (1999), structure functions of order $p>1$ can be calculated by only taking those increments into account for which the two points $x$ and $x+r$ lie on different sides of a shock. If $\delta x \ll r \ll d$, where $\delta x$ is the shock width, the probability that a shock is crossed for an arbitrary increment is equal to $r n / L=r / d$. The $p$ th-order structure function can thus be calculated as

$$
\left\langle|\delta u|^{p}\right\rangle=\left\langle\Delta u^{p}\right\rangle_{s} \frac{r}{d},
$$

where $\Delta u$ is the shock amplitude, taken to be positive by definition, and

$$
\left\langle\Delta u^{p}\right\rangle_{s}=\frac{1}{n} \sum_{i=1}^{n} \Delta u_{i}^{p}
$$

is the average over all shocks. The expression $\Delta u^{p}$ should be read as $(\Delta u)^{p}$. For simplicity, we omit the parentheses. The velocity increment $\delta u$ is always negative over a shock. The third-order structure function can thus be written as

$$
\left\langle\delta u^{3}\right\rangle=-\left\langle\Delta u^{3}\right\rangle_{s} \frac{r}{d}
$$

It is straightforward to derive the Burgers equation analogue of 'the four-fifths law' of Kolmogorov (1941a). Under the assumption of statistical homogeneity and stationarity the following equation is easily derived:

$$
\frac{\partial}{\partial r}\left\langle\delta u^{3}\right\rangle=-12 \epsilon+6 v \frac{\partial^{2}}{\partial r^{2}}\left\langle\delta u^{2}\right\rangle+6\langle\delta u \delta f\rangle .
$$

After integration of (1.5) the two last terms can be neglected if $\delta x \ll r \ll L_{f}$, and we obtain

$$
\left\langle\delta u^{3}\right\rangle=-12 \epsilon r
$$

which was given by Weinan \& Eijnden (1999) and Falkovich \& Sreenivasan (2006). Combining (1.4) and (1.6) we find

$$
\left\langle\Delta u^{3}\right\rangle_{s}=12 \epsilon d,
$$


a relation which was derived by Weinan \& Eijnden (1999). The pth-order structure function can now be written as

$$
\left\langle|\delta u|^{p}\right\rangle=K_{p} \epsilon^{p / 3} d^{p / 3-1} r,
$$

where

$$
K_{p}=12^{p / 3} \frac{\left\langle\Delta u^{p}\right\rangle_{s}}{\left\langle\Delta u^{3}\right\rangle_{s}^{p / 3}}
$$

are non-dimensional prefactors that can be calculated from the probability density function of the shock amplitude $\Delta u$, if that is known. The kinetic energy spectrum can be calculated as the Fourier transform of $-\left\langle\delta u^{2}\right\rangle / 4$. Using the theory of generalised Fourier transforms (Lighthill 1959) we obtain

$$
E(k)=\frac{K_{2}}{2 \pi} \epsilon^{2 / 3} d^{-1 / 3} k^{-2} .
$$

The $k^{-2}$-spectrum was derived by Burgers (1948) in the one-dimensional case and by Kuznetsov (2004) in the three-dimensional case, without including the parametric dependence on $\epsilon$ and $d$. An interesting property of the expressions (1.8) and (1.10) is that they are invariant under superposition of two fields, which follows from the fact that both (1.2) and (1.4) fulfil this type of invariance. As discussed in Frisch (1995), Landau (Landau \& Lifshitz 1987) made the objection against Kolmogorov (1941b) that structure functions cannot be universally dependent on $\epsilon$ and $r$, since such a law would not be invariant under superposition of two fields. This objection cannot be raised against (1.8) and (1.10), since the prefactors $K_{p}$ will adjust in such a way that this invariance is fulfilled.

Recently, Augier, Mohanan \& Lindborg (2019) showed that similar scaling relations are valid for two-dimensional shallow water wave turbulence as for forced Burgers turbulence. For shallow water wave turbulence a distinction should be made between structure functions involving increments of the longitudinal and transverse velocity components, just as in the case of two- or three-dimensional incompressible turbulence. Augier et al. (2019) showed that the ratio between the longitudinal and transverse structure function of a particular order can be determined from the condition that the velocity step at a shock is confined to the shock normal component. It was also shown that the shock width scales as

$$
\delta x \sim \frac{v}{(\epsilon d)^{1 / 3}} .
$$

It can be argued that (1.11) also holds for Burgers turbulence. We will now consider the case of three-dimensional shock-dominated acoustic turbulence

\section{Scaling relations for shock-dominated acoustic turbulence}

We consider an ideal three-dimensional homogeneous isotropic acoustic field in a domain with volume $\mathcal{V}$. We assume that the velocity field is irrotational and that all entropy production in the field is confined to weak shocks, which we consider as smooth surfaces whose total area we denote by $A$. Since it is assumed that the shocks are weak we can also assume that they interact weakly (Apazidis \& Eliasson 2018) and cross each other without strong reflections, just as weak shallow water wave shocks (Augier et al. 2019). Their radius of curvature will therefore be larger than the 


\section{E. Lindborg}

characteristic distance between them. We define the linear mean distance between the shocks as $d \equiv L / n$, where $L$ is the length of a straight line segment passing through the domain and $n$ is the number of shocks that the segment crosses. By the assumptions of isotropy and homogeneity, all segments of all straight lines will give the same value of $d$, provided that sufficiently many shocks are crossed. A structure function of a scalar flow variable can be calculated just as in the one-dimensional case, as the average over a line segment of a moment of the increment - for example, the density increment $\delta \rho=\rho(\boldsymbol{x}+\boldsymbol{r})-\rho(\boldsymbol{x})$, where $\boldsymbol{r}$ is the separation vector. The density structure function of order $p$ can thus be calculated as

$$
\left\langle|\delta \rho|^{p}\right\rangle_{l}=\left\langle\Delta \rho^{p}\right\rangle_{s} \frac{r}{d}
$$

where \langle\rangle$_{l}$ is the line average, $\Delta \rho$ is the density step at a shock, which we take to be positive by definition, and \langle\rangle$_{s}$ is the average over all shocks that are crossed by the segment.

A structure function can also be calculated as a domain average, which we denote by \langle\rangle , without any subscript. By only taking those increments into account for which $\boldsymbol{r}$ crosses a shock, we find

$$
\left\langle|\delta \rho|^{p}\right\rangle=\frac{1}{\mathcal{V}} \int_{\mathcal{V}}|\delta \rho|^{p} \mathrm{~d} V=\frac{1}{\mathcal{V}} \int_{A} \Delta \rho^{p} r \cos \theta \mathrm{d} A,
$$

where $\theta$ is the angle between $\boldsymbol{r}$ and the shock normal unit vector, $\boldsymbol{n}$, defined in such a way that $\theta \in[-\pi / 2, \pi / 2]$. By the assumption of isotropy $\theta$ can be regarded as a random variable with probability density $|\sin \theta| / 2$. We thus find

$$
\left\langle|\delta \rho|^{p}\right\rangle=\langle\cos \theta\rangle_{\theta}\left\langle\Delta \rho^{p}\right\rangle_{A} \frac{r A}{\mathcal{V}}=\frac{1}{2}\left\langle\Delta \rho^{p}\right\rangle_{A} \frac{r A}{\mathcal{V}},
$$

where

$$
\left\langle\Delta \rho^{p}\right\rangle_{A}=\frac{1}{A} \int_{A} \Delta \rho^{p} \mathrm{~d} A
$$

is the average over the total shock area and

$$
\langle f(\theta)\rangle_{\theta}=\frac{1}{2} \int_{-\pi / 2}^{\pi / 2}|\sin \theta| f(\theta) \mathrm{d} \theta .
$$

Evidently, the domain average (2.3) must be equal to the line average (2.1) and $\left\langle\Delta \rho^{p}\right\rangle_{A}$ must be equal to $\left\langle\Delta \rho^{p}\right\rangle_{s}$. Therefore, we must also have

$$
\frac{\mathcal{V}}{A}=\frac{1}{2} d,
$$

a relation which will be used in the following. In this context it may be relevant to point out that the corresponding relation for an isotropic field in two dimensions is $\mathcal{A} / \mathcal{L}=2 d / \pi$, where $\mathcal{A}$ is the domain area and $\mathcal{L}$ is the total shock length. In an anisotropic field the mean crossing distance, $d$, will be different for different lines. A regular grid of squares with side $a$ has $\mathcal{A} / \mathcal{L}=a / 2$. A line which is aligned with the grid has $d=a$, while a line at $45^{\circ}$ angle to the grid (and not crossing the corners of the squares) has $d=a / \sqrt{2}$. 


\section{A note on acoustic turbulence}

The velocity step at a shock, $\Delta u$, which we take to be positive by definition, is confined to the shock normal component. A shock crossing velocity increment can thus be resolved as $\delta \boldsymbol{u}=\delta u_{r} \boldsymbol{e}_{r}+\delta u_{\theta} \boldsymbol{e}_{\theta}$, where $\delta u_{r}=-\cos \theta \Delta u, \delta u_{\theta}=\sin \theta \Delta u, \boldsymbol{e}_{r}=\boldsymbol{r} / r$ and $\boldsymbol{e}_{\theta}$ is orthogonal to both $\boldsymbol{e}_{r}$ and $\boldsymbol{n} \times \boldsymbol{e}_{r}$. The structure function $S_{m n}=\left\langle\left|\delta u_{r}\right|^{m}\left|\delta u_{\theta}\right|^{n}\right\rangle$ can be calculated as

$$
S_{m n}=2\left\langle|\cos \theta|^{m+1}|\sin \theta|^{n}\right\rangle_{\theta}\left\langle\Delta u^{m+n}\right\rangle_{A} \frac{r}{d}
$$

If $m$ and $n$ are integers we can also calculate the structure function $\left\langle\delta u_{r}^{m} \delta u_{\theta}^{n}\right\rangle$, which is equal to zero if $n$ is odd, equal to $S_{m n}$ if both $m$ and $n$ are even and equal to $-S_{m n}$ if $m$ is odd and $n$ is even, since $\delta u_{r}$ is always negative over a shock (Augier et al. 2019). The second- and third-order structure functions are of particular interest:

$$
\begin{gathered}
\left\langle\delta u_{r}^{2}\right\rangle=\left\langle\delta u_{\theta}^{2}\right\rangle=\frac{1}{2}\left\langle\Delta u^{2}\right\rangle_{A} \frac{r}{d}, \\
\left\langle\delta u_{r}^{3}\right\rangle=\frac{3}{2}\left\langle\delta u_{r} \delta u_{\theta}^{2}\right\rangle=-\frac{2}{5}\left\langle\Delta u^{3}\right\rangle_{A} \frac{r}{d} .
\end{gathered}
$$

The radial increment, $\delta u_{r}$, is, of course, nothing else than the longitudinal increment, $\delta u_{L}$, of standard incompressible turbulence theory (Frisch 1995). However, the increment $\delta u_{\theta}$ should not be confused with an arbitrary transverse increment $\delta u_{T}$, since the subscript $\theta$ indicates a specific transverse direction in the vicinity of a shock. To obtain $\left\langle\delta u_{L}^{m} \delta u_{T}^{n}\right\rangle$ one should multiply $\left\langle\delta u_{r}^{m} \delta u_{\theta}^{n}\right\rangle$ by $\int_{0}^{2 \pi} \sin ^{n} \phi \mathrm{d} \phi / 2 \pi$. For example, $\left\langle\delta u_{T}^{2}\right\rangle=\left\langle\delta u_{\theta}^{2}\right\rangle / 2$, and the first equality in (2.8) is therefore consistent with

$$
\left\langle\delta u_{L}^{2}\right\rangle=\frac{\mathrm{d}}{\mathrm{d} r}\left(r\left\langle\delta u_{T}^{2}\right\rangle\right),
$$

which holds for a three-dimensional irrotational isotropic velocity field (see appendix A). From (2.9) we also get $\left\langle\delta u_{L} \delta u_{T}^{2}\right\rangle=\left\langle\delta u_{L}^{3}\right\rangle / 3$, which is only a kinematic consequence of the shock structure, since - generally - there are two independent invariants of the third-order tensor structure function of an irrotational isotropic field. This is shown in appendix A.

Assuming that the shocks for most of their lifetime are in a quasistationary state we can use the shock relations for an ideal gas together with the entropy equation to relate $\left\langle\Delta u^{3}\right\rangle_{A}$ to the mean entropy production over the shocks. For weak shocks, the jump of a flow quantity can be expanded in terms of the shock strength, defined as $z=\left(p_{2}-p_{1}\right) / p_{1}$, where $p_{1}$ and $p_{2}$ are the pressures before and after the shock, respectively. The jumps in velocity, density, temperature and specific entropy can be expanded as (Whitham 1970, p. 176)

$$
\begin{gathered}
\frac{\Delta u}{c_{1}}=\frac{z}{\gamma}+O\left(z^{2}\right), \\
\frac{\Delta \rho}{\rho_{1}}=\frac{z}{\gamma}+O\left(z^{2}\right), \\
\frac{\Delta T}{T_{1}}=\frac{\gamma-1}{\gamma} z+O\left(z^{2}\right), \\
\frac{\Delta S}{c_{v}}=\frac{\gamma^{2}-1}{12 \gamma^{2}} z^{3}+O\left(z^{4}\right),
\end{gathered}
$$




\section{E. Lindborg}

where $c_{1}$ is the speed of sound before the shock and $\gamma=c_{p} / c_{v}$ is the ratio of the specific heats at constant pressure and constant volume. The jump in specific entropy can also be calculated by integrating the entropy equation, which we write in conservative form as

$$
\frac{\partial}{\partial t}(\rho S)+\nabla \cdot(\rho \boldsymbol{u} S)=\frac{k}{T} \nabla^{2} T+\frac{\varepsilon}{T},
$$

where $k$ is the thermal conductivity and $\varepsilon$ is the kinetic energy dissipation rate per unit volume. In the frame of reference where the shock is at rest, the partial time derivative can be neglected, since we assume that the shock is in a quasistationary state. The advective term can be written as $\nabla \cdot(\rho \boldsymbol{u} S)=Q \partial_{x} S$, where $Q$ is the mass flux per unit area over the shock measured in the rest frame of the shock and $x$ is the local shock normal coordinate. The equation can be integrated as (Whitham 1970, p. 189)

$$
\Delta S=\frac{1}{Q} \int_{0}^{\delta x}\left\{\frac{k}{T^{2}}\left(\frac{\partial T}{\partial x_{i}}\right)^{2}+\frac{\varepsilon}{T}\right\} \mathrm{d} x,
$$

where two terms including $\partial_{x} T$ evaluated at the boundaries of the shock have been neglected. Since the Mach number is assumed to be close to unity, and density fluctuations are assumed to be small, we can make the approximation $Q \approx \rho_{0} c_{0}$, where $\rho_{0}$ and $c_{0}$ are background reference values of the density and the speed of sound, respectively, which we take as the mean values over the whole field. Using $c^{2}=\gamma R T$, where $R$ is the ideal gas constant, replacing $c$ by $c_{0}, \rho$ by $\rho_{0}$, and averaging over the total shock area, we obtain

$$
\langle\Delta S\rangle_{A} \approx \frac{1}{A} \int_{A} \int_{0}^{\delta x} \frac{R \gamma}{c_{0}^{3}}\left\{\frac{\kappa c_{p}}{T}\left(\frac{\partial T}{\partial x_{i}}\right)^{2}+\frac{\varepsilon}{\rho_{0}}\right\} \mathrm{d} x \mathrm{~d} A,
$$

where $\kappa=k /\left(\rho c_{p}\right)$ is the thermal diffusivity. Assuming that the entropy production is confined to the shocks, the domain of integration can be extended to include the whole field, and we get

$$
\langle\Delta S\rangle_{A} \approx \frac{R \gamma}{c_{0}^{3}}(\epsilon+\chi) \frac{\mathcal{V}}{A}=\frac{R \gamma}{c_{0}^{3}}(\epsilon+\chi) \frac{d}{2},
$$

where $\epsilon \equiv\left\langle\varepsilon / \rho_{0}\right\rangle$ is the mean kinetic energy dissipation rate per unit mass and

$$
\chi \equiv \kappa c_{p}\left\langle\frac{1}{T}\left(\frac{\partial T}{\partial x_{i}}\right)^{2}\right\rangle
$$

We can now estimate $\left\langle\Delta u^{3}\right\rangle_{A}$ to leading order in $z$ by taking the cube of (2.11), replacing $c_{1}$ by $c_{0}$, averaging over all shocks, and using (2.14) and (2.18),

$$
\left\langle\Delta u^{3}\right\rangle_{A} \approx \frac{6}{\gamma+1}(\epsilon+\chi) d,
$$

which is analogous to relation (1.7) derived by Weinan \& Eijnden (1999) for Burgers turbulence. Inserting this expression into (2.9) we obtain

$$
\left\langle\delta u_{r}^{3}\right\rangle=-C(\epsilon+\chi) r,
$$




\section{A note on acoustic turbulence}

where $C$ is a positive constant of the order of unity. In the weak shock limit we obtain $C=12 / 5(\gamma+1)$, giving $C=9 / 10$ for a monatomic gas and $C=1$ for a diatomic gas. Equation (2.21) is similar to the 'four-fifths law', $\left\langle\delta u_{r}^{3}\right\rangle=-(4 / 5) \epsilon r$, (Kolmogorov 1941a) for incompressible turbulence and the corresponding law (1.6) for Burgers turbulence (Weinan \& Eijnden 1999; Falkovich \& Sreenivasan 2006), with the difference that $\epsilon$ is replaced by $\epsilon+\chi$.

From (2.20) we can conclude that the shock amplitude scales as $\Delta u \sim(\epsilon+\chi)^{1 / 3} d^{1 / 3}$ and that a structure function of order $p>1$ scales as $\left\langle\left|\delta u_{r}\right|^{p}\right\rangle \sim(\epsilon+\chi)^{p / 3} d^{p / 3-1} r$. The structure functions $\langle\delta \boldsymbol{u} \cdot \delta \boldsymbol{u}\rangle=\left\langle\delta u_{r}^{2}\right\rangle+\left\langle\delta u_{\theta}^{2}\right\rangle$ and $\left\langle\left(c_{0} \delta \rho / \rho_{0}\right)^{2}\right\rangle$ are of particular interest, as are $\left\langle\delta u_{r} \delta \boldsymbol{u} \cdot \delta \boldsymbol{u}\right\rangle$ and $\left\langle\delta u_{r}\left(c_{0} \delta \rho / \rho_{0}\right)^{2}\right\rangle$. Replacing the local density $\rho_{1}$ with $\rho_{0}$ in (2.12) and the local speed of sound in (2.11) with $c_{0}$ will only give rise to alterations of $O\left(z^{2}\right)$ on the right-hand sides. To leading order in $z$ we thus find

$$
\begin{gathered}
\left\langle\left(c_{0} \delta \rho / \rho_{0}\right)^{2}\right\rangle=\langle\delta \boldsymbol{u} \cdot \delta \boldsymbol{u}\rangle \sim(\epsilon+\chi)^{2 / 3} d^{-1 / 3} r, \\
\left\langle\delta u_{r}\left(c_{0} \delta \rho / \rho_{0}\right)^{2}\right\rangle=\left\langle\delta u_{r} \delta \boldsymbol{u} \cdot \delta \boldsymbol{u}\right\rangle=-\frac{5}{3} C(\epsilon+\chi) r
\end{gathered}
$$

where the expression for $\left\langle\delta u_{r}\left(c_{0} \delta \rho / \rho_{0}\right)^{2}\right\rangle$ is similar to the Yaglom (1949) relation for the third-order velocity-scalar structure function of incompressible turbulence. The second-order velocity structure function is associated with kinetic energy and the density structure function with the energy form which has been referred to as 'acoustic potential energy' (Lighthill 1978, p. 13). These two forms of energy are equipartitioned in a linear acoustic wave. Quite interestingly, to leading order in $z$, equipartition also holds for a field of weak shocks, and if the third-order structure functions are supposed to be associated with energy fluxes, the relation (2.23) indicates that kinetic and potential energy fluxes are equipartitioned, just as in shallow water wave turbulence (Augier et al. 2019). The kinetic and potential energy spectra scale as

$$
E_{K}(k)=E_{P}(k) \sim(\epsilon+\chi)^{2 / 3} d^{-1 / 3} k^{-2} .
$$

The non-dimensional prefactors which we have omitted in (2.22) and (2.24) can be expressed in such a way that they can be determined from the constant $C$ and the probability density function of $\Delta u$. Thereby, the expressions are invariant under superposition of two fields, just as the corresponding expressions for Burgers turbulence.

To compare the magnitudes of $\epsilon$ and $\chi$, we can use (2.11) and (2.13) to estimate both of them in terms of $z$ :

$$
\begin{gathered}
\epsilon \sim\left(\frac{4}{3} v+v_{b}\right) \frac{c_{0}^{2}}{\gamma^{2} \delta x d}\left\langle z^{2}\right\rangle_{A}, \\
\chi \sim \kappa \frac{c_{0}^{2}(\gamma-1)}{\gamma^{2} \delta x d}\left\langle z^{2}\right\rangle_{A},
\end{gathered}
$$

where $v_{b}=\mu_{b} / \rho_{0}$, with $\mu_{b}$ being the bulk viscosity. Defining a Prandtl number as $\operatorname{Pr} \equiv\left(4 v / 3+v_{b}\right) / \kappa$, we see that $\chi \sim \epsilon$ if $\operatorname{Pr} \sim 1$. If this is the case, the shock strength can be estimated as $z \sim(\epsilon d)^{1 / 3} / c_{0}$, the Mach number, $M=\sqrt{1+(\gamma+1) z / 2 \gamma}$, as $M \sim 1+(\epsilon d)^{1 / 3} / c_{0}$ and the shock width as in (1.11). The lifetime of a shock can be estimated as $\tau \sim \delta x^{2} / v \sim v /(\epsilon d)^{2 / 3}$ and the ratio between the partial time derivative and the advective term in the entropy equation (2.15) can thus be estimated as $\delta x / \tau c_{0} \sim(\epsilon d)^{1 / 3} / c_{0}$, motivating the assumption of quasistationarity. 


\section{E. Lindborg}

\section{Conclusions}

We showed that similar scaling relations hold for an acoustic field that is dissipated by weak shocks as for forced Burgers turbulence, with the difference that $\epsilon$ should be replaced by $\epsilon+\chi$, where $\chi$ is associated with entropy production due to heat conduction. In all likelihood, the ratio $\chi / \epsilon$ is Prandtl-number-dependent and as long as the Prandtl number is of the order of unity, $\chi$ is of the same order as $\epsilon$. Apart from $\gamma$, the third-order structure function of acoustic turbulence therefore depends on two parameters - unlike Burgers turbulence, where there this is a single parameter dependence. This is not very surprising. In the full equations, describing an acoustic field, the pressure term is the agent of exchange between kinetic and internal energy, and these two forms of energy are of equal importance. In the Burgers equation, on the other hand, the pressure term is absent and internal energy is irrelevant. From a principal point of view, the replacement of $\epsilon$ by $\epsilon+\chi$ makes a big difference. The third-order structure function laws of incompressible turbulence and Burgers turbulence are connected with the notion of a constant energy flux through scales, which is equal to $\epsilon$. It remains a theoretical challenge to investigate in what way the quantity $\epsilon+\chi$ may be linked to an energy flux. From an experimental point of view the replacement of $\epsilon$ by $\epsilon+\chi$ is of less importance if $\operatorname{Pr} \sim 1$. Let us assume that we set out to test the prediction of Kadomtsev \& Petviashvili (1973) that an acoustic field is always dissipated by shocks if the Reynolds number is sufficiently large, and that we do this by generating a random acoustic field in a chamber with reflecting walls, using loudspeakers whose total input power is $P=10^{-3} \mathrm{~W} \mathrm{~kg}^{-1}$. Let us further assume that shocks are formed with a mean distance $d=0.1 \mathrm{~m}$. In a stationary state, we have $\epsilon=P$, and given the relations derived in this paper we can estimate the relative pressure change over a shock as $z \sim 10^{-4}$, the Mach number as $M \sim 1+10^{-4}$, the shock velocity amplitude as $\Delta u \sim 5 \mathrm{~cm} \mathrm{~s}^{-1}$, the shock width as $\delta x \sim 0.5 \mathrm{~mm}$ and the Reynolds number as $\operatorname{Re}=\Delta u d / v \sim d / \delta x \sim 200 \gg 1$. Indeed, it would be an experimental challenge to produce an image of a field of such weak shocks, but it does not seem to be insurmountable. Likewise, the art of direct numerical simulations has developed into a stage in which it would be feasible to test the prediction of Kadomtsev \& Petviashvili (1973) by making a full Navier-Stokes simulation of a randomly forced acoustic field. It is the hope of the author that the present note will stimulate research along these lines.

\section{Acknowledgements}

The author would like to thank G. Falkovich for commenting on an early version of the manuscript. Two anonymous reviewers are acknowledged for useful comments.

\section{Appendix A. Invariants of structure functions}

In this appendix we derive relation (2.10) and show that there are two invariants of the third-order tensor structure function of an isotropic irrotational velocity field. The isotropic second-order structure function can be written as

$$
\left\langle\delta u_{i} \delta u_{j}\right\rangle=e_{i} e_{j}\left\langle\delta u_{L}^{2}\right\rangle+s_{i j}\left\langle\delta u_{T}^{2}\right\rangle,
$$

where $\boldsymbol{e}=\boldsymbol{r} / r$ and $s_{i j}=\delta_{i j}-e_{i} e_{j}$. By isotropy, $\left\langle\delta u_{L}^{2}\right\rangle$ and $\left\langle\delta u_{T}^{2}\right\rangle$ are functions of $r$. By applying the irrotational condition $\epsilon_{i j k} \partial_{j}\left\langle\delta u_{k} \delta u_{l}\right\rangle=0$ and using $\partial_{i} n_{j}=s_{i j} / r$ we obtain

$$
\frac{1}{r} \epsilon_{i l k} e_{k}\left(\left\langle\delta u_{L}^{2}\right\rangle-\frac{\mathrm{d}}{\mathrm{d} r}\left(r\left\langle\delta u_{T}^{2}\right\rangle\right)\right)=0,
$$




\section{A note on acoustic turbulence}

from which (2.10) follows. The third-order tensor structure function can be written as

$$
\left\langle\delta u_{i} \delta u_{j} \delta u_{k}\right\rangle=2\left(D_{i j k}+D_{j k i}+D_{k i j}\right),
$$

where $D_{i j k}=\left\langle u_{i} u_{j} u_{k}^{\prime}\right\rangle$. Unprimed and primed quantities indicate positions $\boldsymbol{x}$ and $\boldsymbol{x}+\boldsymbol{r}$, respectively. In (A 3) we have used that $\left\langle u_{i}^{\prime} u_{j}^{\prime} u_{k}\right\rangle=-\left\langle u_{i} u_{j} u_{k}^{\prime}\right\rangle$ by isotropy and $\left\langle u_{i}^{\prime} u_{j}^{\prime} u_{k}^{\prime}\right\rangle=$ $\left\langle u_{i} u_{j} u_{k}\right\rangle$ by homogeneity. Since the velocity field is irrotational we can write $D_{i j k}=$ $\left\langle\partial_{i} \phi \partial_{j} \phi \partial_{k}^{\prime} \phi^{\prime}\right\rangle$, where $\phi$ is the velocity potential. By homogeneity we have

$$
D_{i j k}=\frac{\partial B_{i j}}{\partial r_{k}}
$$

where $B_{i j}=\left\langle\partial_{i} \phi \partial_{j} \phi \phi^{\prime}\right\rangle$. The irrotational condition $\epsilon_{i j k} \partial_{j} D_{l m k}=0$ is clearly fulfilled by (A 4). By isotropy we can write $B_{i j}=e_{i} e_{j} a(r)+s_{i j} b(r)$, where $a(r)$ and $b(r)$ are two scalar functions. It is quite clear that $a(r)$ and $b(r)$ are generally independent of each other since there is no further constraint on $B_{i j}$. A little bit of algebra gives

$$
\begin{gathered}
\left\langle\delta u_{L} \delta u_{L} \delta u_{L}\right\rangle=6 \frac{\mathrm{d} a}{\mathrm{~d} r}, \\
\left\langle\delta u_{L} \delta u_{T} \delta u_{T}\right\rangle=2 r^{2} \frac{\mathrm{d}}{\mathrm{d} r}\left(\frac{b}{r^{2}}\right)+\frac{4 a}{r} .
\end{gathered}
$$

We conclude that the third-order tensor structure function of an isotropic irrotational field has two invariants.

\section{References}

Apazidis, N. \& Eliasson, V. 2018 Shock Focusing Phenomena. Springer.

Augier, P., Mohanan, A. V. \& Lindborg, E. 2019 Shallow water wave turbulence. J. Fluid Mech. 874, 1169-1196.

Bouchaud, J. P., Mezard, M. \& Parisi, G. 1995 Scaling and intermittency in Burgers turbulence. Phys. Rev. E 52 (4, A), 3656-3674.

Burgers, J. M. 1948 A mathematical model illustrating the theory of turbulence. Adv. Appl. Mech. 47, 95-114.

Falkovich, G. \& Meyer, M. 1996 Two-dimensional acoustic turbulence. Phys. Rev. E 54 (4), 4431-4434.

Falkovich, G. \& Sreenivasan, K. R. 2006 Lessons from hydrodynamic turbulence. Tech. Rep. 163. IAEA.

FRISCH, U. 1995 Turbulence. Cambridge University Press.

Kadomtsev, B. \& Petviashvili, V. 1973 On acoustic turbulence. Dokl. Akad. Nauk SSSR 208, 794-796.

Kolmogorov, A. N. 1941 a Dissipation of energy in locally isotropic turbulence. Dokl. Akad. Nauk SSSR 32, 16-18.

Kolmogorov, A. N. $1941 b$ The local structure of turbulence in viscous fluid for very large Reynolds number. Dokl. Akad. Nauk SSSR 30, 299-303.

Kuznetsov, E. A. 2004 Turbulence spectra generated by singularities. JETP Lett. 80 (2), 83-89.

Landau, L. D. \& Lifshitz, E. M. 1987 Fluid Mechanics. Pergamon Press.

Lighthill, J. 1978 Waves in Fluids. Cambridge University Press.

Lighthill, M. J. 1959 Introduction to Fourier Analysis and Generalised Functions. Cambridge University Press.

L'vov, V. S., L'vov, Y., Newell, A. C. \& Zakharov, V. 1997 Statistical description of acoustic turbulence. Phys. Rev. E 56, 390-405. 


\section{E. Lindborg}

Weinan, E. \& EIJnden, E. V. V. 1999 Asymptotic theory for the probability density functions in Burgers turbulence. Phys. Rev. Lett. 83 (13), 2572-2575.

Weinan, E., Khanin, K., Mazel, A. \& Sinai, Y. 1997 Probability distribution functions for the random forced Burgers equation. Phys. Rev. Lett. 78 (10), 1904-1907.

Whitham, G. B. 1970 Linear and Nonlinear Waves. Wiley.

Yaglom, A. M. 1949 On the local structure of a temperature field in a turbulent flow. Dokl. Akad. Nauk SSSR 69, 743-746.

Zakharov, V. E. \& Sagdeev, R. Z. 1970 Spectrum of acoustic turbulence. Sov. Phys. Dokl. 15, 439-441. 